\title{
Detection of radiation treatment of spices using Electron Spin Resonance Spectroscopy technique
}

\author{
A A Khan ${ }^{1} \&$ M K Shahid ${ }^{2, *}$ \\ ${ }^{1}$ Department of Chemistry, Government Post Graduate College, Haripur 22620, KPK, Pakistan \\ ${ }^{2}$ Research Institute of Environment \& Biosystem, Chungnam National University, Yuseong-gu, Daejeon \\ 34134, Republic of Korea. \\ *E-mail address: mkbutt2000@gmail.com
}

Received 07 January 2021; Revised 29 April 2021; Accepted 10 May 2021

\begin{abstract}
Use of ionising radiation for commercial sterilisation to increase the hygienic quality of spices is increasing worldwide. Among several detection methods, Electron Spin Resonance (ESR) spectroscopy is one of the reliable techniques for irradiated foods containing some hard and dried parts. Spices are therefore candidates of such a method and radiation treated spices can be detected. The purpose of the present work was to identify the radiation treatment of spices like cinnamon, cardamom, ginger and rosemary using the sensitive technique of ESR spectroscopy. The spice samples were irradiated to 5, 10, 15 and 20 kilogray (kGy) or unirradiated and the samples were dried in vacuum oven and were placed into quartz tubes for ESR measurement. On measurements, the unirradiated samples were found to show the ESR-signal at g-value of 2.004 that is typical in case of plant foods containing cellulose. Whereas, the irradiated samples showed a formation of a new paramagnetic structure that appeared in the form of a triplet-signal, attributed to radiation-induced cellulose radical. The clear difference between the nature of ESR signals in case of unirradiated and irradiated samples provided the evidence of the radiation treatment of spices samples. It is therefore concluded that radiation treatment of spices can be identified using sensitive ESR spectroscopy during a storage period of three to ten months.
\end{abstract}

Keywords: electron beam, food irradiation, food safety, free radical, hygienic quality, spices.

\section{Introduction}

Spices are widely used ingredients in a number of food preparations in various countries of the world (Thanushree et al. 2019). The use of spices in food industry has tended to increase for the production of "convenience foods"; hence these are important commodities in international trade. Spices are generally considered an expensive food ingredient. Pakistan is among the countries, like Brazil, China, India, Tanzania and Spain, which are the largest exporters of spices (Akhtar et al. 2020; Zachariah 2020). Spices, therefore, represent an important share in the national economy of Pakistan. 
Many spices are grown in developing countries and usually the hygienic quality cannot be maintained due to lack of proper handling from harvest to the country of export. Spices, like other agricultural commodities, generally contain microbial populations in the range of $10^{4}-10^{8}$ colony-forming units per gram (CFU g-1) (Minarovičová et al. 2018). Moulds are present as dominant micro flora along with pathogenic bacteria in spices. A relatively high incidence of Clostridium perfringenes and a wide variety of non-spore forming bacteria like coliforms are frequent (Aguilera et al. 2005). Similarly, mould flora like Aspergillus glaucus group, Aspergillus niger and Penicillum spp. are usually more prevalent (Sucheta, Chaturvedi \& Yadav 2019). The pathogen-contaminated spices have been identified as one of the major causes of foodborne diseases (Van Doren et al. 2013).

There are several decontamination methods in use but processing of foods with ionising radiation is the most versatile treatment. It is safe, efficacious, environment friendly and energy efficient. This process can eliminate potentially pathogenic non-spore forming bacteria from suspected food products without affecting the quality of food. Radiation decontamination of spices and many other dry food ingredients is a viable alternative to less effective processes and has a great application potential both in developing and industrialized countries (Chmielewski \& Migdał 2005; Molnár et al. 2018).

OrganizationssuchasWorldHealthOrganisation (WHO), International Atomic Energy Agency (IAEA), National Food Processors Association (NFPA), Food and Drug Administration (FDA) of USA and several university-based food research institutes have endorsed irradiation as a technique to preserve food from microbial spoilage and to maintain the microbiological safety (Ananthi et al. 2019; Khatun et al. 2021). A number of benefits are associated with the use of this technology and different dose limits for achieving different kinds of benefits, like enhancing hygiene quality of food, spoilage reduction and shelf-life extension have been proposed and recommended (World Health Organization 1995).
Food irradiation technology is now accepted and has been regulated in a number of countries throughout the world (Farkas \& Mohácsi-Farkas 2011). The detection of radiation treatment of spices is essential for administrative control, for assisting international trade and for reinforcing buyer confidence on radiation treatment. Therefore, the detection of irradiated food is of great significance to implement quality compliance at all levels. Because of importance of detection of irradiated foods, several countries as well as international organizations, such as European Committee for Standardisation (CEN) have recommended a number of analytical methods for identification of irradiated foods (Stefanova, Vasilev \& Spassov 2010). These include Electron Spin Resonance (ESR), also called as Electron Paramagnetic Resonance (EPR) spectroscopy of foods containing bones, cellulose or sugar, DNA comet assay of food containing DNA-material, thermo-luminescence of foods contaminated with silicate minerals or gas-chromatographic analysis of foods containing lipid-derived radiolytic products (Delincée 2002; Aoudé-Werner et al. 2020).

Detection of irradiated foods using different methods is being continuously investigated. An appropriate detection technique should analyse the specific radiation impact, which is relative to the dosage and should not be influenced by processing factors and storage conditions. In the past, ESR has been proposed as a method for identification of several irradiated food items including spices (Aleksieva \& Yordanov 2018). In the present study, ESR spectroscopy for the detection of irradiated spices was tested.

The prime objective of present work is to investigate the presence of the radiationinduced free radicals in irradiated spices such as cinnamon, cardamom, rosemary and whole ginger by ESR spectroscopy. This will assist food professionals in the routine screening of unirradiated and irradiated samples as well as to investigate the stability of ESR signals at different intervals of time during post-irradiation storage of these spices. 


\section{Materials and Methods}

Irradiation apparatus and irradiation of spice samples

The spice samples were purchased from the local market in Karlsruhe, Germany and were stored at room temperature. Small amounts of different spices (approx. $100 \mathrm{~g}$ ) were packed into little polyethylene bags for the purpose of irradiation.

The samples were treated with ionizing radiation using a 10 mega electron-volt $(\mathrm{MeV})$ electron beam (Circe III linear accelerator, Thomson-CSF Linac, St. Aubin, France) having a dose rate of $10^{8} \mathrm{~Gy} / \mathrm{s}$ (in pulse). Radiochromic films, GAF DM-1260 (International Speciality Products, Wayne, USA) (Li et al. 2000) were used for the measurement of the radiation doses by evaluating them at $405 \mathrm{~nm}$ for change in optical density with the help of a filter photometer (Ciba Corning Halstead, Essex, UK). The radiation doses applied to the spice samples were as per the guidelines of International Consultative Group on Food Irradiation. For each of the spice under study four doses of radiation $(5,10,15$, and $20 \mathrm{kGy})$ were used, beside the control (unirradiated) samples. After irradiation, the small packages of samples were stored at room temperature until EPR measurements.

\section{Sample preparations for ESR analysis}

Both the controlled and treated samples of cinnamon, cardamom, rosemary and ginger were prepared according to the protocol of European Committee for Standardisation (CEN) for cellulose containing foods (EN1787 2000). The sample materials of all spices were dried in vacuum oven at $40^{\circ} \mathrm{C}$ for $3 \mathrm{~h}$. About $200 \mathrm{mg}$ was taken from each spice sample in EPR quartz tubes (length; $150 \mathrm{~mm}$, internal diameter; $4 \mathrm{~mm}$ and wall-thickness; $0.1 \mathrm{~mm}$ ) for placing them to microwave cavity of the instrument for recording the spectra.
ESR spectrometer and spectrometric settings for ESR analysis

The measurements of the ESR - spectra were made using Bruker EMS 104 EPR Analyzer fitted with a double rectangular cavity. In order to ensure the reproducibility and stability of measurements, the instrument calibration was carried out daily before each measurement. This was achieved for checking the response and setting of EPR spectrometer by means of solid 1, 1-diphenyl-2-picrylhydrazyl (DPPH), a strong pitched standard (Bruker). For ensuring the accurate and reproducible position of the sample in the cavity of instrument, a positioning template was used for each sample present in the ESR quartz tubes and desired height was adjusted through parameter settings of the instrument.

As the samples of the spices under consideration contained cellulose, the spectrometric settings were in accordance with protocol of CEN specified for the food containing cellulosic material (EN1787 2000). Therefore, the operating conditions used in this study were: microwave frequency of 9.8 $\mathrm{GHz}$ and microwave power of $0.8 \mathrm{~mW}$; centre field of $348 \mathrm{mT}$ (3480 Gauss) and sweep width of $20 \mathrm{mT}$ (200 Gauss); modulation frequency of $50 \mathrm{kHz}$ and modulation amplitude of $0.8 \mathrm{mT}$ (8 Gauss) with time constant (filter t.c) of 40.96 $\mathrm{ms}$ and sweep time $42 \mathrm{~s}^{\text {sweep }}{ }^{-1} ; 10$ sweeps spectrum $^{-1}$; receiver gain between $45-50 \mathrm{~dB}$; spectral record taken at room temperature.

\section{Results and Discussion}

After the measurements, the recorded ESR spectra of unirradiated and irradiated cinnamon samples are as shown in Fig. 1, while for cardamom and ginger samples, the spectra are shown in Fig. 2 and 3. The comparison of intensities of ESR triplet signals regarding post-irradiation stability for the spices like cinnamon and rosemary is shown in Fig. 4. 

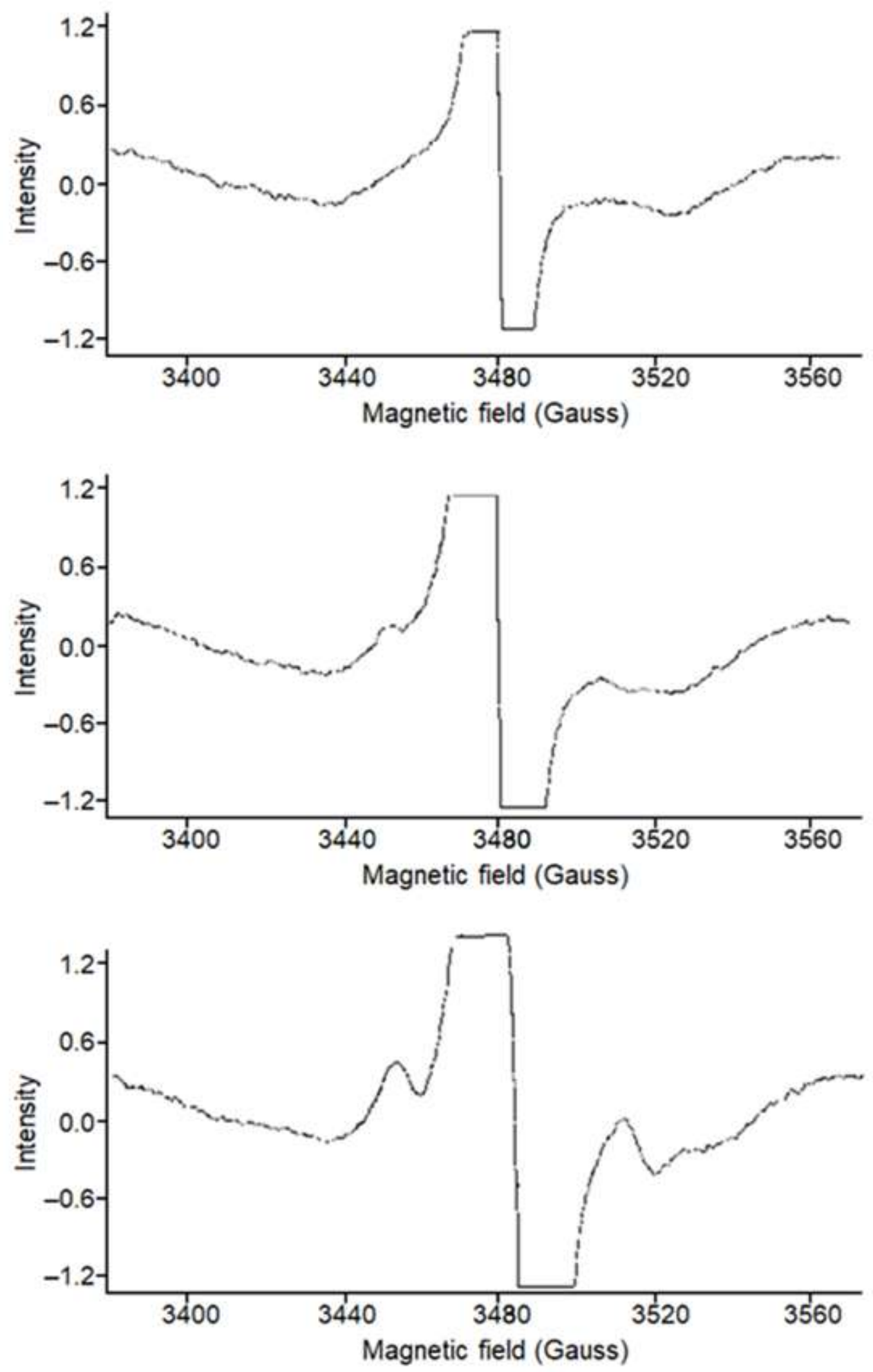

Fig. 1 ESR spectra of cinnamon after storage of 90 days at room temperature; unirradiated sample (top), sample irradiated to $5 \mathrm{kGy}$ (middle), samples irradiated to $20 \mathrm{kGy}$ (bottom). 
Assessment of the EPR signals

A single central signal with g-value of 2.004 was observed in the spectra, which is typical to food samples containing cellulose both in case of unirradiated and irradiated samples (Fig. 1-3; spectra at top in each case). The intensity of this signal was observed to be high in irradiated samples. The irradiated samples were observed to have a pair of satellite lines one to the left (lower field) and the other to the right (higher field) of the central signal. This pair of lines along with central signal gave rise to the triplet signal (a central signal with two satellite lines on both sides) and this attributes to the radiation-induced cellulose radical. This signal is considered to be radiation-specific and is a marker of radiation treatment (Ahn et al. 2013). A spacing of $6 \mathrm{mT}$ (60 Gauss) has been noted between the right and left signal. As formation of cellulose radical considered being formed due to the radiation treatment, the presence of such satellite lines (pair of lines) on either side of the central signal provided the evidence for radiation treatment of the spice samples, which already contained the crystalline cellulosic material (Fig. 1-3; spectra in middle and at bottom). The radiationinduced side peaks were found to be consistent with reported studies on irradiation of different food additives (Chiappinelli et al. 2019).

\section{Stability of EPR signals for irradiation detection}

All the samples of irradiated spices showed the triplet signal (one central and two lines on either side) due to radiation-induced cellulose radicals. The stability and intensities of these radicals have been shown to be dependent upon the nature of the material (here spices) as well as on the environmental influences (Karakirova \& Yordanov 2020). The post-irradiation stability of radiation-specific signals or at least the presence of the triplet-signal in analysis seems to be important for administrative control of the radiation processing. However, during some studies on spices like coriander (Sezer, Kaplan \& Sayin 2017), clove (Gaspar et al. 2019) curry leaf, allspice (El-Sayed \& Youssef 2019), paprika (Molnár et al. 2018) and peppers, the radiationinduced cellulose radical could be detected at least several weeks after treatment and thus provided the evidence for identification of radiation treatment.

In the present study, the samples of the spices from cinnamon, cardamom, rosemary and ginger have shown clear triplet signals for different applied doses of radiation in the range of 5-20 kGy. These spices have been shown to be promising candidates of the detection of radiation treatment

Fig. 1 Shows three spectra of cinnamon; spectrum related to unirradiated sample (at top), sample related to radiation dose of $5 \mathrm{kGy}$ (in middle) and sample related to dose of $20 \mathrm{kGy}$ (at bottom). The unirradiated and irradiated samples could nicely be distinguished in order to screen out treated and un-treated samples. Different radiation doses, which were delivered to the samples of spices, could be differentiated based on intensities of radiation-specific triplet signals. It was noted that even after the passage of about 10 months, the signals could be recorded. Hence, for the long period of 10 months or more, detection of radiation treatment of cinnamon was practically possible using the sensitive technique of ESR spectroscopy.

Fig. 2 shows the spectra of cardamom; spectrum related to unirradiated sample (at top), sample related to radiation dose of $5 \mathrm{kGy}$ (in middle) and sample related to dose of $20 \mathrm{kGy}$ (at bottom). From the examination of spectra, qualitative differentiations between irradiated and unirradiated samples are achieved. The investigations showed the presence of distinct triplet signal in case of the samples irradiated to $20 \mathrm{kGy}$ even after post-irradiation storage of 10 months. This signal was of sufficiently higher in intensity as compared to some other spices and seems to be dependent upon preirradiation conditions of the sample under study. 

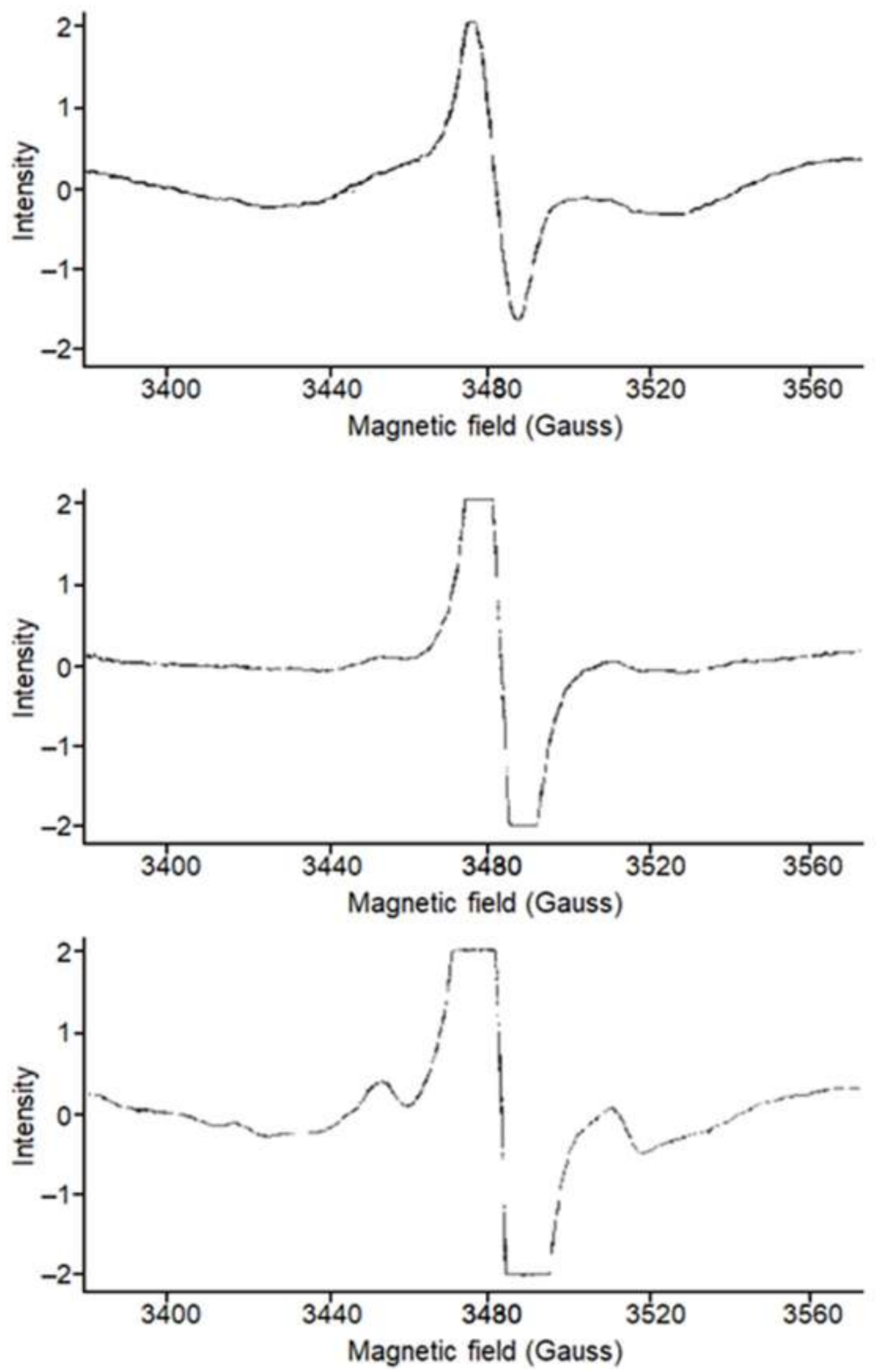

Fig. 2 ESR spectra of cardamom during post-irradiation storage at room temperature; unirradiated sample (top), sample irradiated to $5 \mathrm{kGy}$ (middle), samples irradiated to $20 \mathrm{kGy}$ (bottom). 
Fig. 3 shows the spectra of ginger; spectrum (at top) related to unirradiated sample and spectrum (at bottom) related to radiation dose of $20 \mathrm{kGy}$. In case of ginger, the radiation doses of 5 and $10 \mathrm{kGy}$ could not be detected and radiation dose of other than $20 \mathrm{kGy}$ could not be detected after a post-irradiation period of about three months. This study was able to show the possibility of discrimination between unirradiated and irradiated samples of $20 \mathrm{kGy}$ up to storage period of 3 months. In the figure, the spectrum at the bottom ( $20 \mathrm{kGy})$ is labelled showing radiation-induced triplet signal and pair of lines on either side of central signal.

Fig. 4 shows plot of post-irradiation storage period versus ESR signal intensity. This indicates a comparison between postirradiation stability of signals from the samples of cinnamon and rosemary. The ESR measurements were recorded at intervals of 15, 100 and 300 days. The signal intensities in case of cinnamon were found to be greater than rosemary at all intervals. Up to 300 days (10 months), reasonable amount of radiationinduced cellulose radicals with easily registered
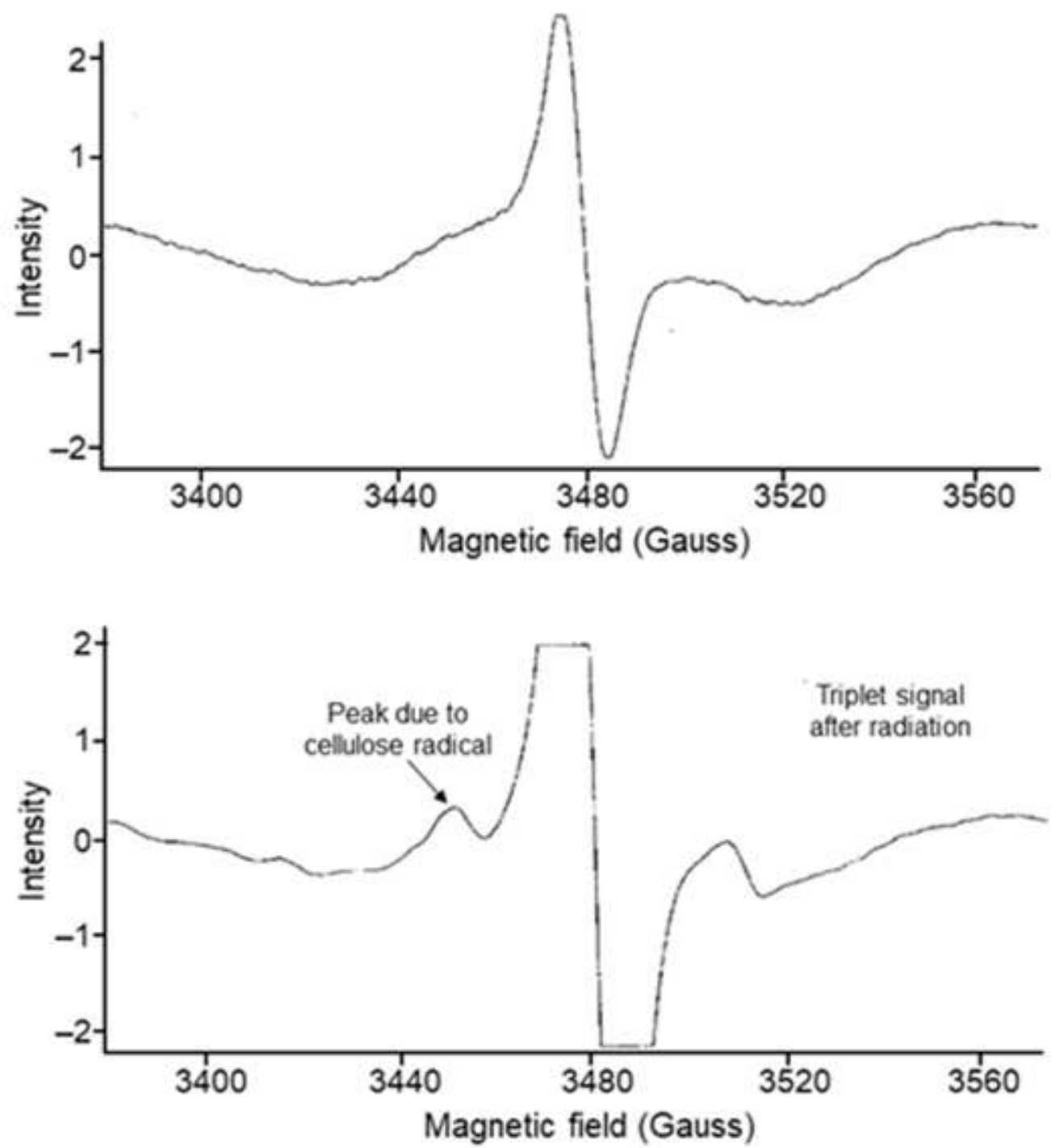

Fig. 3 ESR spectra of whole ginger after storage of 90 days at room temperature; a: (top) Unirradiated sample, b: (bottom) sample irradiated to $20 \mathrm{kGy}$. 


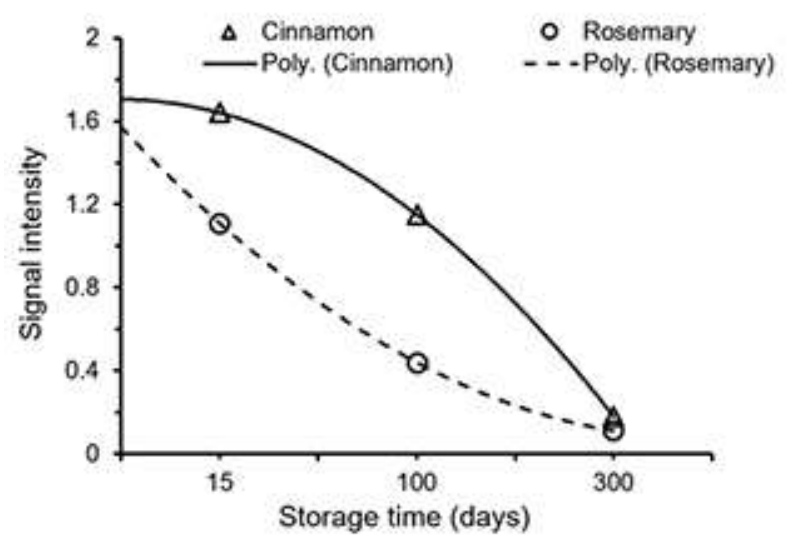

Fig. 4 Comparison between irradiated (20 kGy) cinnamon and rosemary regarding postirradiation stability of ESR signals up to storage of 10 months.

signal intensities was identified in both cases i.e., cinnamon and rosemary. If this trend is extrapolated to more storage time under similar conditions, it is expected that detection of radiation treatment of both spices would be possible up to 11 months or more.

Spices, like coriander, juniper, cumin, black pepper, chilli, lovage and marjoram have shown intense ESR signals, and the signals remained relatively stable up to three months after irradiation (Oliveira, Cruz-Tirado \& Barbin 2019). Similarly, referring to a past study regarding ginger, the absence of cellulose radicals in ESR signal in case of low doses of radiations seems to be due to relatively higher moisture contents in ginger, because it was reported that moisture could decrease the intensity of the signal by a factor of about 10 (Stachowicz, Burlinska \& Michalik 1998).

In some studies on paprika, white peppers (Ha, Li \& Wang 2011), nutmeg (Calucci et al. 2003) and ginger (Choi et al. 2004), the EPR spectroscopy was not found to be ideal, since the free radicals formed were short lived and could not be distinguished from intrinsic free radicals already present in the controlled samples. However, our investigations on ginger samples showed a different trend and it was noted that samples irradiated to higher doses (20 kGy or above) could be differentiated from the unirradiated samples even after three months of post-irradiation storage of samples. Hence, it is concluded that ESR might be a potential technique for qualitative identification of irradiated spice samples, and for evaluation of the indigenous doses in the spice samples. ESR can also be applied to evaluate the radio-stability of food components including preservatives (e.g., butylated hydroxytoluene) and vitamins (e.g., biotin, riboflavin, thiamine hydrochloride, thiamine mononitrate, pyridoxine, and folic acid)(Yu \& Cheng 2008).

The samples of cinnamon, cardamom and rosemary irradiated in the range of 5-20 kGy could be discriminated from the unirradiated samples up to a storage period of about 10 months (or more). Hence, identification of radiation treatment of these spices is possible for a reasonable storage period in the context of trade and commercialization. However, the whole ginger samples, irradiated to $20 \mathrm{kGy}$ could only be differentiated from unirradiated samples. The ESR triplet signals from samples of ginger, which were irradiated at 5, 10, and $15 \mathrm{kGy}$, could not be detected. Keeping in view the results obtained, ESR spectroscopy can be recommended for effective detection of irradiation over a considerable period of storage of spices. The potential areas for future research and development include the assessment of high-dose irradiation impact on food quality and the use of integrated processes such as combination of irradiation and other techniques.

\section{Acknowledgement}

We are grateful to Dr. H. Delincée of Federal Research Centre for Nutrition, Karlsruhe, Germany for providing facilities to complete this research work. One of the authors, Ashfaq A. Khan is grateful to the Ministry of Science and Technology, Pakistan for S\&T fellowship.

\section{References}

Aguilera, Milton O, Patricia V S, Blas M \& Ana M S D G 2005. "Prevalence and Characterization of Clostridium Perfringens from Spices 
in Argentina." Anaerobe 11 (6): 327-34. doi:10.1016/j.anaerobe.2005.05.003.

Ahn, Jae J, Kashif A, Hyun K K \& Joong H K 2013. "Electron Spin Resonance Spectroscopy for the Identification of Irradiated Foods with Complex ESR Signals." Food Analytical Methods 6 (1): 301-8. doi:10.1007/s12161-0129440-4.

Akhtar, Saeed, Muhammad R, Iqra N, Yun Y G, Amir I, Majid H \& Kashif A 2020. “Risk Assessment of Aflatoxins and Selected Heavy Metals through Intake of Branded and NonBranded Spices Collected from the Markets of Multan City of Pakistan." Food Control 112 (January). Elsevier: 107132. doi:10.1016/j. foodcont.2020.107132.

Aleksieva, Katerina I., \& Nicola D Y 2018. “Various Approaches in EPR Identification of GammaIrradiated Plant Foodstuffs: A Review." Food Research International 105 (October 2017): 1019-28. doi:10.1016/j.foodres.2017.11.072.

Ananthi, V, G Siva Prakash, Soon W C, Balasubramani R, Dinh D N, Dai-Viet N V \& Duong D L et al. 2019. “Enhanced Microbial Biodiesel Production from Lignocellulosic Hydrolysates Using Yeast Isolates." Fuel 256: 115932. doi:https://doi.org/10.1016/j. fuel.2019.115932.

Aoudé-Werner, Dalal, Irene S, Véronique Z \& Florent K 2020. "Identification of Bleached and Irradiated Walnuts and Hazelnuts by ESR Spectroscopy." Radiation Physics and Chemistry 173 (March). Elsevier Ltd: 108882. doi:10.1016/j.radphyschem.2020.108882.

Calucci, Lucia, Calogero P, Maurizio Z, Antonella C, Silvia G, Franco S, Sabrina T \& Luciano G 2003. "Effects of $\gamma$-Irradiation on the Free Radical and Antioxidant Contents in Nine Aromatic Herbs and Spices." Journal of Agricultural and Food Chemistry 51 (4): 92734. doi:10.1021/jf020739n.

Chiappinelli, Andrea, Michele M, Michele T, Gabriele T, Giuliana M \& Antonio Eugenio Chiaravalle. 2019. "Identification of X-RayIrradiated Hazelnuts by Electron Spin Resonance (ESR) Spectroscopy." European Food Research and Technology 245 (10). Springer Berlin Heidelberg: 2323-29. doi:10.1007/s00217-019-03349-2.
Chmielewski, Andrzej G \& Wojciech M 2005. "Radiation Decontamination of Herbs and Spices." Nukleonika 50 (4): 179-84.

Choi, In-duck, Myung-woo B, Joong-ho K \& Dong-ho K 2004. "An Application of PhotoStimulated Luminescence (PSL) and Electron Spin Resonance (ESR) Analysis for the Irradiated Spicy Vegetables." Food Science and Biotechnology 13 (5): 646-50.

Delincée \& Henry 2002. "Analytical Methods to Identify Irradiated Food - A Review." Radiation Physics and Chemistry 63 (3-6): 455-58. doi:10.1016/S0969-806X(01)00539-4.

Doren, Jane M. Van, Karen P N, Mickey P, Laura G, Hannah G L \& Kathy L G 2013. “Foodborne Illness Outbreaks from Microbial Contaminants in Spices, 1973-2010." Food Microbiology 36 (2). Elsevier Ltd: 456-64. doi:10.1016/j.fm.2013.04.014.

El-Sayed, Samah M \& Ahmed M Y 2019. "Potential Application of Herbs and Spices and Their Effects in Functional Dairy Products." Heliyon 5 (6). Elsevier Ltd: e01989. doi:10.1016/j.heliyon.2019.e01989.

EN1787. 2000. "Foodstuffs - Detection of Irradiated Food Containing Cellulose by ESR Spectroscopy." European Committee of Standardization (CEN). Brussels, Belgium.

Farkas, József \& Csilla M F 2011. "History and Future of Food Irradiation." Trends in Food Science and Technology 22 (2-3): 121-26. doi:10.1016/j.tifs.2010.04.002.

Gaspar, Elvira M, José C S, Pedro M P S, João P T \& Abel J S C V 2019. “Gamma Irradiation of Clove: Level of Trapped Radicals and Effects on Bioactive Composition." Journal of the Science of Food and Agriculture 99 (4): 166874. doi:10.1002/jsfa.9351.

Ha, Yi M, Wei M L \& Feng W 2011. “Application of ESR Spectroscopy to Identify and Estimate Original Dose in Irradiated Cumin and White Pepper." European Food Research and Technology 233 (4): 625-30. doi:10.1007/ s00217-011-1556-z.

Karakirova, Yordanka \& Nicola D Y 2020. “Time Dependence of the EPR and Optical Spectra of Irradiated Crystal Sugar." Radiation Physics and Chemistry 168 (March 
2019). Elsevier Ltd: 108569. doi:10.1016/j. radphyschem.2019.108569.

khatun, Mst A, Md Razzak, Md Afzal H, Md Ashikur R, Ruhul A K \& Roksana H 2021. “Gamma Radiation Application to Rice: Reduced Glycemic Index in Relation to Modified Carbohydrate Observed in FTIR Spectra." Current Research in Food Science 4: 11-17. doi:https://doi.org/10.1016/j.crfs.2020.12.002.

Li, Zhongying, Dezhi W, Dahua C, Shouyong P, Lu Z \& Keqin S 2000. "A Study of Dosimetry Characteristics of GAF DM-1260 Radiochromic Films." Radiation Physics and Chemistry 57 (2): 103-13. doi:10.1016/S0969806X(99)00345-X.

Minarovičová, Jana, Tereza C, Eva K, Anneluise M, Janka L, Peter S \& Tomáš K 2018. “CultureIndependent Quantification of Pathogenic Bacteria in Spices and Herbs Using Real-Time Polymerase Chain Reaction." Food Control 83: 85-89. doi:10.1016/j.foodcont.2016.12.025.

Molnár, Helga, Ildikó B V, Erzsébet B, Zsuzsanna C, Sándor F, Rita T F, Nóra A \& András S 2018. "The Effect of Different Decontamination Methods on the Microbial Load, Bioactive Components, Aroma and Colour of Spice Paprika." Food Control 83: 131-40. doi:10.1016/j.foodcont.2017.04.032.

Oliveira, Marciano M, Cruz-Tirado J P \& Douglas F B 2019. "Nontargeted Analytical Methods as a Powerful Tool for the Authentication of Spices and Herbs: A Review." Comprehensive Reviews in Food Science and Food Safety 18 (3): 670-89. doi:10.1111/1541-4337.12436.

Sezer, Özgür M, Necati K \& Ulku S 2017. “ESR Analysis of Natural and Gamma Irradiated Coriander (Coriandrum Sativum L.) Seeds." Radiation Effects and Defects in Solids 172 (11-12): 815-23. doi:10.1080/10420150.2017.1 402329 .

Stachowicz W, Burlinska G \& Michalik J 1998. “EPR Detection of Foods Preserved with
Ionizing Radiation." Radiation Physics and Chemistry 52 (1-6): 157-60. doi:10.1016/ S0969-806X(98)00066-8.

Stefanova, Rayna, Nikola V V \& Stefan L S 2010. "Irradiation of Food, Current Legislation Framework, and Detection of Irradiated Foods." Food Analytical Methods 3 (3): 22552. doi:10.1007/s12161-009-9118-8.

Sucheta, Kartikey C \& Sudesh K Y 2019. "Ultrasonication Assisted Salt-Spices Impregnation in Black Carrots to Attain Anthocyanins Stability, Quality Retention and Antimicrobial Efficacy on HotAir Convective Drying." Ultrasonics Sonochemistry 58 (June). Elsevier: 104661. doi:10.1016/j.ultsonch.2019.104661.

Thanushree M P, Sailendri D, Yoha K S, Moses J A \& Anandharamakrishnan C 2019. "Mycotoxin Contamination in Food: An Exposition on Spices." Trends in Food Science and Technology 93 (September 2018): 69-80. doi:10.1016/j.tifs.2019.08.010.

World Health Organization. 1995. Safety and Nutritional Adequacy of Irradiated Food. World Health Organization. doi:10.1016/ s0969-806x(96)90041-9.

Yu, Liangli Y \& Zhihong C 2008. "Application of Electron Spin Resonance (ESR) Spectrometry in Nutraceutical and Food Research." Molecular Nutrition \& Food Research 52 (1). John Wiley \& Sons, Ltd: 62-78. doi:https:// doi.org/10.1002/mnfr.200700395.

Zachariah T J 2020. "Major Indian Spices- An Introspection on Variability in Quality." Journal of Spices and Aromatic Crops 29 (7): 1-20. doi:10.25081/josac.2020.v29.i1.6331.

Zanardi, Emanuela, Augusta C \& Enrico N 2018. "New Insights to Detect Irradiated Food: An Overview." Food Analytical Methods 11 (1). Food Analytical Methods: 224-35. doi:10.1007/s12161-017-0992-1. 